http://jmscr.igmpublication.org/home/ ISSN (e)-2347-176x ISSN (p) 2455-0450

crossref DOI: https://dx.doi.org/10.18535/jmscr/v8i3.36

\title{
Interesting Case Presentation of Bickerstaff Brainstem Encephalitis with Overlapping Guillain Barre Syndrome
}

\author{
Authors \\ Dr R Vishnupriya ${ }^{1}$, Dr B Vinu' ${ }^{2}$, Prof. Dr V.R. Mohan Rao ${ }^{3}$ \\ ${ }^{1}$ Postgraduate, Department of General Medicine, Chettinad Hospital \&Research Institute, Chettinad \\ Academy of Research and Education \\ ${ }^{2}$ Assistant Professor, Department of General Medicine, Chettinad Hospital \& Research Institute, Chettinad \\ Academy of Research and Education \\ ${ }^{3}$ Professor, Department of General Medicine, Chettinad Hospital \& Research Institute, Chettinad Academy \\ of Research and Education
}

\begin{abstract}
Bickerstaff brainstem encephalitis is a neurological disease characterized by ophthalmoplegia, ataxia with disturbed consciousness which is a variant of Guillain Barre syndrome. Here we describe a atypical presentation of Bickerstaff brainstem encephalitis with overlapping Guillain Barre syndrome features.

Keywords: Guillain Barre syndrome (GBS), Bickerstaff brainstem encephalitis (BBE), Raised protein, Peripheral nerve lesion.
\end{abstract}

\section{Introduction}

Bickertstaff brainstem encephalitis is a rare variant of Guillian- barre syndrome which has a geographically variable incidence. Both can be considered as a part of same spectrum of disease with an autoimmune mechanism, triggered by an antecedent infection. It is mainly a clinical diagnosis based on the cardinal features of ophthalmoplegia, ataxia with disturbed consciousness .It also has features of hypereflexia, babinski's sign which differentiates it from Guillain Barre syndrome. We report a 49 yr old woman who presented with lower limb ascending type of weakness with facial and bulbar palsy suggesting GBS and ataxia, ophthalmoplegia with hyperreflexia and babinski's sign suggesting BBE but without any disturbance in consciousness which is considered to be a rare presentation.

\section{Case Report}

49 Year old woman with no previous medical history and comorbidities presented to OPD with the complaints of sudden onset of dysphagia and dysarthria with unsteadiness of gait and difficulty in using lower limbs for 1 week duration. She had no similar complaints in the past.

On examination, patient was conscious, oriented, afebrile, pale with stable vitals and normal single breath count. Neurological examination revealed normal tone and reduced power of $3 / 5$ in both lower limbs (Proximal>Distal) and hypereflexia, Plantar was extensor. Cranial nerve examination revealed bilateral sluggish pupillary response to light with restriction of eye movements in all directions. She was unable to close the eyes tightly with reduced prominence of nasolabial fold bilaterally. She also had decreased movement on 
right palate with gag reflex lost on right side. Cerebellum examination revealed stance ataxia on left side. Sensory examination was normal. At the end of examination, she was found to have bilateral lower limb weakness with bilateral $3^{\text {rd }}$ nerve palsy, bilateral LMN facial nerve palsy, Right $9^{\text {th }}, 10^{\text {th }}$ cranial nerve palsy with ataxia. Other systems examination were normal.

Differential diagnosis of Acute brainstem stroke, Multiple sclerosis, acute disseminated encephalomyelitis were initially considered.

Basic investigations showed Hb-11, TC-14,000, ESR-15.Her renal functions, electrolytes, ECG were all normal. CT Brain done and it was found to be normal.MRI brain with MRA showed no abnormality. MRI whole spine was also found to be normal. Electromyography showed peripheral nerve dysfunction. A lumbar puncture was performed which demonstrated a raised protein levels with no cells.CSF oligoclonal bands was taken to rule out multiple sclerosis which was reported as negative.

The diagnosis of Variant of Guillain Barree syndrome-Bickerstaff brainstem encephalitis overlapping with GBS was considered based on clinical feature of ataxia, ophthalmoplegia and hypereflexia with babinski sign and lower limb weakness, facial and bulbar weakness, CSF Showing acellular picture and raised proteins, Electromyography picture showing peripheral nerve lesion.

The patient was treated with IV immunoglobulins and plasmapharesis. The patient recovered completely after 3 weeks with no neurological deficit.

\section{Discussion}

GBS is an acute severe fulminant polyradiculoneuropathy presenting as a reflex motor paralysis without any sensory disturbances ${ }^{1}$.Ascending paralysis with facial weakness is present in more than half of the case ${ }^{2}$. Lower cranial nerves are frequently involved, which may be mistaken for brainstem ischaemia ${ }^{3}$. One of the variant of GBS is Bickerstaff brainstem encephalitis which was described by Edwin Bickerstaff as 'grave syndrome with beningn prognosis' ${ }^{6}$. Usual presentation is ophthalmoplegia, ataxia with disturbed consciousness. Odaka et al clarified the neurological features of 62 cases of BBE which includes disturbed consciousness, hypereflexia, babinski's sign. Other features includes facial weakness and bulbar palsy. He classified patients with BBE presenting with symmetrical limb weakness as BBE with overlapping GBS. ${ }^{4}$

Miller fischer syndrome, GBS and BBE can be considered as part of same spectrum of disease with an autoimmune mechanism triggered by an antecedent infection. The diagnostic criteria for BBE are 1)Progressive symmetrical ophthalmoplegia, ataxia by 4 weeks 2)Conscious disturbance or pyramidal signs(hyper reflexia) 3)limb strength of 5 or 4 . Some other clinical features of $\mathrm{BBE}$ are characterized as facial weakness, bulbar palsy and long tract sensory disturbances. ${ }^{5}$ The diagnosis of $\mathrm{BBE}$ is by high index of clinical suspicion. Brain abnormality on MRI scan is found only in $30 \%$ of patients and anti-GQ1 Ab in $66 \%$ based on few studies. But absence of anti-GQ1 Ab and normal MRI do not exclude the diagnosis. ${ }^{7}$

In many cases of BBE, antecedent pathogens have been identified which gives evidence that antiganglioside antibodies work via molecular mimicry with infectious agents. ${ }^{21}$ They includes herpes simplex virus, cytomegalovirus, $E B V$, varicella-zoster virus, measles virus, Mycoplasma pneumonia and Campylobacter jejuni enteritis ${ }^{8}$.

Various treatment modalities are used for BBE which includes steroids, IV Igs and plasmapharesis ${ }^{9}$. Our patient presented with lower limb ascending type of weakness with facial and bulbar weakness suggesting GBS and ataxia, ophthalmoplegia with hyperreflexia and babinski's sign suggesting BBE but without any disturbance in consciousness with a cellular raised proteins in CSF and EMG showing peripheral 
nerve lesion. So BBE overlapping GBS is considered in our patient based on clinical picture. Our patient was treated with IV immunoglobulins and plasmapharesis. And she dramatically responded to the treatment without any deficit.

\section{Conclusion}

This case is reported for its rare presentation of a Bickerstaff brainstem encephalitis with overlapping features. Although the condition is rare, the favourable response to medical treatment warrant early diagnosis and prompt treatment.

\section{References}

1. Harrison's Manual of Internal Medicine 20th Edition

2. Odaka M, Yuki N, Yamada M, et al. Bickerstaff's brainstem encephalitis: clinical features of 62 cases and a subgroup associated with Guillain-Barre syndrome. Brain 2003;126(Pt 10):2279-90 [PubMed] [Google Scholar]

3. Bickerstaff E, Cloake PCP. Mesencephalitis and rhombencephalitis. BMJ 1951;2:77-81 [PMC free article] [PubMed] [Google Scholar]

4. Bickerstaff ER. Brain-stem encephalitis; further observations on a grave syndrome with benign prognosis. BMJ 1957;1:13847 [PMC free article] [PubMed] [Google Scholar]

5. Ito M. Bickerstaff's brainstem encephalitis and Fisher syndrome form a continuous spectrum: clinical analysis of 581 cases. Journal of Neurology 2008. 255:674-82.
6. Clinical deterioration in Bickerstaff's brainstem encephalitis caused by overlapping Guillain-Barré syndrome. Susuki K, Johkura K, Yuki N, Kuroiwa YJ Neurol Sci. 2003 Jul 15; 211(1-2):8992.[PubMed] [Ref list]

7. Yuki N. Anti-ganglioside antibody and neuropathy: review of our research. J Peripher Nerv Syst 1998;3:3-18 [PubMed] [Google Scholar]. 